\title{
Establishment and Validation of The Axillary Lymph Node Burden Using Cone-Beam Computerized Tomography and Ultrasound-Based Prediction Models in T1-2 Breast Cancer Patients
}

\section{Shen Chen}

Sun Yat-sen University Cancer Center

Jiao Li

Sun Yat-sen University Cancer Center

Peiqiang Cai

Sun Yat-sen University Cancer Center

Tiebao Meng

Sun Yat-sen University Cancer Center

Weimei Ma

Sun Yat-sen University Cancer Center

Jieting Chen

Sun Yat-sen University Cancer Center

Chuanmiao Xie

Sun Yat-sen University Cancer Center

Shengting Pei

Sun Yat-sen University Cancer Center

Chunyan Zhou

Sun Yat-sen University Cancer Center

Lizhi Liu

Sun Yat-sen University Cancer Center

$\mathrm{Ni} \mathrm{He}$

Sun Yat-sen University Cancer Center

Yaopan Wu ( $D$ wuyp@sysucc.org.cn )

Sun Yat-sen University Cancer Center Jianye Liang

Sun Yat-sen University Cancer Center

Research Article 
Keywords: breast cancer, Cone-beam breast computed tomography, Axillary ultrasound, lymph node burden, prediction factor

Posted Date: January 21st, 2021

DOI: https://doi.org/10.21203/rs.3.rs-137791/v1

License: (1) This work is licensed under a Creative Commons Attribution 4.0 International License. Read Full License 


\section{Abstract}

Background: This study aimed to develop and validate models to preoperatively predict the risk of the lymph node (LN) burden based on the Z0011 clinical trial to assist breast cancer surgical decisionmaking.

Methods: Data on 1394 consecutive patients who presented at Sun Yat-sen University Cancer Center for Cone-beam breast computerized tomography (CBBCT) examinations between April 3, 2019, and July 17, 2020, were retrospectively collected. 387 patients who met the inclusion criteria were included and randomly divided into training and validation cohorts. Clinical-pathological information of all patients was recorded, and images were reinterpreted in this study. A bidirectional stepwise method followed by multi-variable analysis was used to incorporate preoperative features and build optimal model sets with

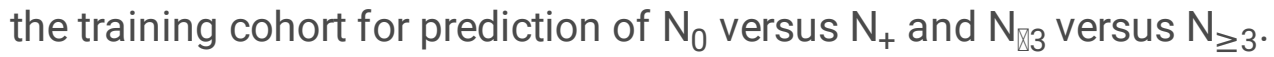

Results: The ROC curves of two models were generated with the training cohort, and their calibration abilities were estimated using 1000 bootstrap resamples. The bias-corrected C-index of the models were $0.779(95 \% \mathrm{Cl}, 0.752-0.793)$ in model one and $0.809(95 \% \mathrm{Cl}, 0.794-0.833)$ in model two for the training cohort. Decision curves and clinical impact curves were plotted to evaluate prediction performance for further clinical application. Delong's test showed comparable performance of both cohorts.

Conclusions: Our models were developed as reliable and noninvasive tools for the preoperative prediction of nodal status, and we hope that they can serve as useful tools for the early planning of treatment strategies for breast cancer patients.

\section{Introduction}

Breast cancer is one of the most commonly diagnosed malignancies in women, ranking first in terms of mortality among women and seriously affecting female health worldwide (1). Because of its rapid progression and insidious symptoms in the early stage of biological invasion, breast cancer should be closely monitored with current advanced technology (2). The outcome of axillary lymph node dissection (ALND) plays an important role in the formulation and implementation of breast cancer regimens, including postoperative radiotherapy and chemotherapy (3). It is traditionally used for patients with metastatic sentinel lymph node (SLN) during surgery. However, a large number of previous reports based on the Z0011 clinical trial verified the advantages of forgoing standard ALND, which may cause complications, such as lymphedema, infection, limitations of shoulder motion, major vessel and nerve injury and overtreatment for non-metastatic patients $(4,5)$. Early-stage invasive breast cancer patients with only 1 or 2 SLN metastases can be regarded as having a low burden and receive radiotherapy as a substitute after sentinel lymph node biopsy (SLNB) (6). Compared with ALND, SLNB is mainly applied in early-stage breast cancer patients and has fewer traumatic complications. However, there is a potential risk of false negatives due to the distant metastasis of other regions that are not detected or destruction of the drainage network of patients with a high clinical stage $\left(T_{3-4}\right)(7,8)$. Moreover, patients with early 
breast cancer who have node-negative disease can actually forgo SLNB. Therefore, an accurate preoperative assessment of the lymph node (LN) status can avoid unnecessary traumatic procedures such as ALND and SLNB in the post-Z0011 era (9). Thus, taking these circumstances into account, studies have been undertaken to discover a possible relationship between primary tumor behavior and lymphatic metastasis to aid in reliable preoperative diagnostic evaluation (10).

The application of the textual analysis of radiographic data reveals the heterogeneity within the tumor tissue with a quantitative assessment; however, the accuracy of lesion segmentation restricts its application. Deep learning improves its performance by automatically learning discriminative features from images (11). As a novel approach in the field of radiology, the utility of its clinical application still needs to be verified with more studies. The manual interpretation of medical images still accounts for a large proportion of the actual work. Therefore, prediction models with descriptors on medical images can add value by assisting with $L N$ evaluation.

Cone-beam breast computed tomography (CBBCT), an emerging and promising diagnostic imaging tool, has been broad application currently. Its clinical value has been verified (12) with advantages in the visualization of breast masses and microcalcification with enhancement due to its acquisition of high spatial and contrast resolution images. It has advantages, including the 3D reconstruction of the breast to reduce overlapping glands and increase the clear display of lesions (12). A previous study showed good agreement and repeatability in the assessment of breast density and lesions between readers $(13,14)$. Due to its convenient methods of 3D reconstruction, it can simultaneously display the relationship between vessels and tumors (15). It is time- and cost-saving and provides a more comfortable experience (16). CBBCT achieved comparable diagnostic accuracy to ultrasound $\backslash$ magnetic resonance imaging \mammography; however, its field of view cannot cover the whole breast area, and LN appearance are not visible on its images (14).

Accordingly, axillary ultrasound (AUS), another routine and cost-efficient diagnostic tool, is frequently used in the clinic currently. AUS can visualize detailed axillary structures and provide accurate measurements. However, the diagnosis of nodal metastasis via AUS is operator-dependent and imprecise for low axillary metastatic burden (17). Jackson et al. (18) suggested a false negative rate of $4 \%$ for predicting a high burden of heavy-burden $\mathrm{LNs}(\mathrm{N} \geq 3)$.

Although MRI has high sensitivity for detecting abnormalities, it may be limited by its small ROI size and the subjective identification of the region of interest from $L N$, which is defined by the reader manually (19). Therefore, studies have investigated the combined use of different modalities for $L N$ prediction. Most studies show equivalent values in their constructed models $(20,21)$.

In this study, we analyzed a mixture of quantified and semantic data from CT-derived features of the primary tumor and LN features with AUS to examine their relationship. We believe that the pooled impact of tumor and $\mathrm{LN}$ analysis will improve its prediction. 


\section{Materials And Methods \\ Clinical Data and Participants}

This study used anonymous data and received approval from the Institutional Ethics Committee of Sun Yat-sen University Cancer Center (No. B2019-016) .Written informed consent was obtained from all participating patients in the study. A database of 1328 consecutive patients who presented at Sun Yatsen University Cancer Center for CBBCT examination from January 2019 to July 2020 was retrospectively reviewed. Their clinical records were reviewed, with informed consent obtained from all patients, and only patients initially diagnosed with $T_{1}-T_{2}$ stage invasive breast cancer were included in the study cohort. All patients had no history of additional systematic treatment, including chemotherapy, radiation, or ipsilateral breast surgery. Finally, a total of 387 patients who underwent surgery with SLNB or ALND after examination with histologically proven malignant breast tumors were enrolled (Figure 2). The eligible population was randomly divided into two independent cohorts at a ratio of $70.0 \%(271 / 387)$ and $30.0 \%$ (116/387).

\section{Protocols for Dedicated Cone-Beam Breast CT}

All participants underwent dedicated breast CT examination (KBCT 1000®, Koning Corporation) using a single coil for each breast in the prone position. CBBCT images were acquired for the affected side with precontrast injection, 60 s postcontrast injection, and $120 \mathrm{~s}$ postcontrast injection. For each examination, the injection of contrast enhancement materials was performed under generally accepted standard protocols. lodine contrast agent was injected intravenously with a high-pressure injector at a concentration of $300-370 \mathrm{mg} / \mathrm{ml}$ and 1-2 $\mathrm{ml}$ per kilogram for the injection dose. The total amount did not exceed $100 \mathrm{ml}$ for a single patient. During the injection procedure, close attention was paid to the patient's physical condition. The injection speed was set at 2.0-3.0 ml/s. Optimal scanning parameters were set automatically at a constant voltage of $49 \mathrm{kVp}$ and tube current of 50-160 mA (calculated automatically according to the size and density of the breast). The standard reconstruction mode $\left(1024 \times 1024,0.273 \mathrm{~mm}^{3}\right)$ was selected to reconstruct multiplanar images. Dedicated three-dimensional visualization software was utilized (Visage CS Thin Client/Server, Visage Imaging), and the automatic skin removal option was used to better distinguish lesions from the surrounding gland tissue on 3D images.

\section{Reassessment of Image Parameters}

Two radiologists (with four to seven years of experience) who were blinded to any clinical and pathological information reviewed all images independently. Both noncontrast-enhanced and contrastenhanced CBBCT images were reassessed in consensus. Discrepancies with reassessment were resolved by consulting experts in breast cancer diagnosis (Y.P.W.) to reach a final conclusion. $\triangle \mathrm{CT}$ was computed 
using a formula proposed by Prionas et al. (24): $\Delta \mathrm{HU}=\left(\mathrm{HU}_{\text {lesion }}-\right.$ post $-\mathrm{HU}_{\text {fat }}$ - post $)-\left(\mathrm{HU}_{\text {lesion }}-\right.$ pre- $-\mathrm{HU}_{\text {fat }}$ pre). Distance to the nipple was measured manually on sagittal sections. The relationship between vessels and masses was visualized with specialized three-dimensional reconstruction software. (Figure 3)

AUS images were acquired at our hospital using IU22 (PHILIPS, The Netherlands) and ACUSON S2000 (SIEMENS, Germany) systems with a high-frequency transducer (12 to $15 \mathrm{MHz}$ ). Electronic reports were reviewed, and parameters including primary LN morphology, blood flow type and detection site were evaluated and recorded.

\section{Biopsy and Pathology Assessments}

For each patient undergoing a preoperative CT scan, concordance with subsequent pathology outcomes was determined after surgical excision, including the evaluation of "histological grade and types, number of metastatic LNs, bundle/vascular invasion and expression of molecular markers". Histopathological subtypes of breast cancer were classified as invasive ductal carcinoma (IDC), mixed-type (IDC with other components), and special-type breast cancer. Tumor (nuclear) grade was categorized using the Nottingham grading system as a reference. The expression of estrogen receptor (ER), progesterone receptor (PR), human epidermal growth factor receptor type 2 (HER2), and Ki-67 was determined using IHC. Tumors with more than one positive tumor cell on the basis of the nuclear staining intensity were considered to be ER/PR positive (25). The HER2 staining intensity was scored from 0 to 3 , and tumors with scores of $3+$ or positive results of Fisher's test were considered to exhibit HER2 overexpression. For the Ki-67 expression status, nuclear staining of at least $14 \%$ was considered to indicate a high level of proliferation. All LNs were surgically removed via SLNB/ALND and assessed with postoperative biopsy. The presence of micrometastases or macrometastases on SLNB was indicative of a positive LN status. Isolated tumor cells were classified as node-negative. According to the ACOSOG Z0011 criteria, two positive LNs was an optimal cutoff to select patients who can avoid traumatic axillary dissection surgery. Therefore, outcomes of the $L N$ status were divided into three groups to develop two separate clinical prediction models.

\section{Feature Selection and Statistical Analyses}

Patients who met the inclusion criteria were retrospectively reviewed and treated as independent variables in statistical analysis. Two models were constructed using the LN status as outcome indicators: a) prediction of negativity and metastasis (N0 versus $\mathrm{N}+$ ) and b) low burden and high burden (N $₫ 3$ versus $\mathrm{N} \geq 3$ ). All clinical and pathological primary lesion information was derived from a medical database. Continuous variables were analyzed using Levene's test and the Mann-Whitney test, whereas differences in categorical variables were assessed using the chi-squared test, adjusted chi-squared test or Fisher's exact test. Univariate analysis was performed on the primary feature set to choose model parameters. 
Then, a bidirectional elimination method was applied to modify the model by repeatedly adding in and removing features until an optimal equation was developed $(22,23)$. Finally, multivariable logistic regression analysis was used to select statistically meaningful parameters and fit the best prediction model. Statistical analysis was completed by using IBM SPSS version 26 (IBM, Armonk, NY, USA) and R studio software (version $1.3 \mathrm{https}$ ///rstudio.com/products/rstudio/download/). All statistical hypothesis tests were two-sided, and $p$-values $<0.05$ were considered to indicate significance. The randomization method was used to divide the primary cohort into two separate groups with a ratio of " 0.7 * $n$ ". The analysis of continuous and categorical variables was conducted with SPSS software. Feature selection was conducted using bidirectional stepwise regression with the "mass" package. Plotting of the nomogram, calibration curves and clinical impact curves was performed with the package "rms". Decision curve analysis (DCA) construction was performed by means of library (rmda). The evaluation of the prediction ability of ROC curves was performed with "pROC" bag. The Hosmer-Lemeshow test and Cindex were performed using the "ResourceSelection" and "Hmisc" packages.

\section{Assessment and validation of Prediction Performance}

Receiver operating characteristic curves (ROCs) were plotted to assess the discriminative performance of the two prediction models. Additionally, the predictive ability of the two models was compared between the combinative or independent use of two preoperative imaging modalities. The area under the curve (AUC) of both examinations was computed in both the primary and internal validation cohorts. DeLong's test, as a quantitative method for comparing the AUC of two curves, was calculated for overfitting risk between the training and validation cohorts. The predictive performance of the two models was assessed by calibration curves plotted with 1000 bootstrap samples to evaluate the agreement between the predictions and actual observations. The adjusted C-index was calculated to demonstrate the accuracy of the prediction models with bootstrap correction. Hosmer-Lemeshow tests were used to assess the fitness degree.

\section{Clinical Utility of the Final Model}

Axillary LN metastasis prediction was illustrated with nomograms integrating clinicopathological features to provide explicit models for image-assisted treatment planning.To determine the clinical significance of the final model, decision curve analysis and clinical impact curve analysis were performed.

\section{Results}

\section{Summary of Clinicopathological Characteristics}

The distribution of both the training and validation cohorts is described in Figure 1. A total of 239 (61.8\%) patients had no metastatic LNs in biopsy after surgery, 78 patients had one or two metastases in the LNs, 
and the remaining patients $(n=70)$ had more than three $L N$ metastases confirmed by biopsy. According to the Z0011 clinical trial, patients were divided into the nonmetastatic $(n=172)$, low-risk $(n=48)$, and highrisk $(n=51)$ groups in the training group. The baseline clinical characteristics for both the training and validation cohorts are summarized in Table 1. Detailed descriptive clinical and pathological characteristics and semantic features are listed in Supplementary Tables.

\section{Data Analyses}

On univariate analyses, several factors were found to be significantly related to outcomes, including the CT-reported lesion number, maximum diameter, distance to the nipple, thickened or sunken skin, subcutaneous fat space, invasion of the pectoralis major, and relationship between the vessel and mass and the US-reported number of LNs, maximum LN length and axis, LN shape, blood flow type, and boundary between the cortex and medulla, and the pathology-reported bundle/vascular invasion in both models. In model two, lesion number, and the relationship between the vessel and mass were significantly related to outcomes(Table 2). Clinical and pathological features were selected with a bidirectional selection method to reduce complexity and downsize predictive parameters. The AIC (Akaike information criterion) was applied in model construction (22). Models corresponding to the minimum AIC values were selected for construction. Selected features were ulteriorly fed on to multivariable logistic regression analyses that performed to eliminate factors and identify the most significant predictive factors $(p<0.05)$ with clinical benefits associated with the severity of nodal burden (Table 3 ). The final models are consisted of features that were adjusted by multivariable analyses.

\section{Illustration of the Prediction Models}

Nomograms are straightforward, and our prediction models can be better visualized with the aid of nomograms (Figure 4). Selected CBBCT and AUS morphological features were given scores based on the regression coefficients to establish scoring criteria. Hence, a total score can be calculated for each patient and used to determine the probability of predicted nodal risk.

\section{Diagnostic Performance of Prediction Models}

The prediction ability of models incorporating features selected for both CBBCT and AUS yielded AUCs of 0.884 (95\% Cl: $0.841-0.927)$ and 0.891 (95\% Cl: 0.841-0.940) in the training cohort for models one and two, respectively. Figure $5 \mathrm{~A}$ and $\mathrm{B}$ also shows the detailed performance of the combined prediction models versus the individual prediction ability of CBBCT and AUS. For model one, the AUC decreased to 0.733 for CBBCT and 0.727 for AUS. However, each examination of the prediction of negativity versus metastasis (model 1) and the level of LN burden (model 2) was inferior to that of the combined model. In addition, the ROC curves of the training and validation cohorts with AUC values are shown in Figure $5 \mathrm{C}$ and D. The AUC of the validation cohort decreased to 0.878 (95\% Cl: 0.823- 0.933) for model one and 
0.880 (95\% Cl: $0.836,0.925)$ for model two. Delong's test showed that the validation cohort was comparable to the combined models performance in the training cohort for model one $(p=0.341)$ and model two $(p=0.335)$.

\section{Internal Validation of Prediction Models}

The calibration curve demonstrated good agreement between the performance of the training (Figure 6A) and validation (Figure 6B) sets in model one. Figure $6 \mathrm{C}$ and $\mathrm{D}$ illustrate comparable performance for both groups in the second model. Among these four graphs, diagonal red lines represent perfect performance and are closely fit to the diagonal blue line, which indicates better prediction performance. The biascorrected C-index was $0.79(0.85,0.91)$. The goodness-of-fit analysis with the $\mathrm{HL}$ test resulted in a P value of 0.579 , which indicated that the model fit well. For model two, the bias-corrected C-index was 0.75 $(0.81,0.88)$, whereas the $\mathrm{P}$ value of the $\mathrm{HL}$ test indicating goodness-of-fit was 0.440 .

The decision curve for model one showed good prediction performance in both the training and validation groups in terms of its clinical application. Purple curves (Figure 7) were located above the extreme curves, demonstrating that model one can add more benefit within a large range of risk thresholds. However, model 2 yielded no potential clinical net benefit under the threshold of 0.03 .

Clinical impact curves were also generated to assess the clinical usefulness of the prediction models (Figure 8). It visually showed the estimated numbers of individuals who were deemed high-risk and true positives in the range of 0.01 to 0.07 and 0.075 by using risk models one and two, respectively. Given that the rate of LN metastasis was approximately 0.75 and 0.73 (300/400 and 220/300) in each model, the other 600 and 700 participants might avoid ALND with the surveillance of nodal burden through the clinical application of our risk nomogram.

\section{Discussion}

The noninvasive preoperative prediction of levels of LN metastasis has become increasingly important since it can provide relevant information to assist with surgical and adjuvant radiation therapy planning.

In this study, we evaluated whether preoperative parameters could quantify the risk probability of LN outcomes in patients with early-stage breast cancer. Outcomes comprising the prediction goals were based on the clinical trial Z0011 and the current surgical regimen. To the best of our knowledge, this is the first study to date that applied assessment with CBBCT and AUS for nodal status prediction. It represents an innovative method for the early assessment of $L N$ status based on preoperative evaluation with two widely used modalities in the clinic currently. A large number of previous reports adopted the default quantitative values of the radiomics signature from tumor segmentation to reveal additional information that was invisible to the naked eye $(16,22$, ). However, the process of manual assessing lesions in diagnosis cannot be replaced by other means. 
However, LNs are mostly not present on CBBCT scans unless they are enlarged or situated low. Studies have suggested that there are possible connections between the biological behavior of the primary tumor and its lymphatic spread. CBBCT evaluation of tumor size and the number of lesions is important for the prediction of $L N$ burden (26). Multifocality may lead to an additional total tumor burden in patients compared with unifocal breast cancer. Studies have reported that multifocality may also increase lymphatic positivity and the likelihood of locoregional metastasis (27). Additionally, with reference to the TNM classification, Cabioglu et al. (28) combined the assessment of the largest diameter to provide a more accurate assessment of LN burden with clinical value (29).

The characteristic of the distance to the nipple is often used as a critical factor in assessment prior to performing nipple-sparing mastectomy. In this paper, the measurement of the distance between the nipple and tumor was included in the final model because it can affect regional recurrence. Jordan et al. (30) suggested that a tumor-to-nipple distance of $1 \mathrm{~cm}$ or less was the only significant risk factor for recurrence $(\mathrm{OR}, 13.5833 ; \mathrm{p}=0.0385)$ in univariate analysis. The observation that the distance between breast cancer and the nipple impacts axillary nodal metastases has also been verified by several studies $(31,32)$.Obscure subcutaneous fat space was selected as one component in the construction of our model, and similar findings were reported by Zhang et al. (33) Lymphatic nets within the tumor or inside the fat space were interwoven, allowing subcutaneous metastasis to reach the ipsilateral axilla from the breast parenchyma.

Chang et al. (3) suggested that axillary imaging warrants consideration of at least axillary level I or II to examine lymphadenopathy. A degraded muscular status may be indicative of a shorter time of progression (34). Because the structure of pectoralis muscles is anatomically well visualized on CBBCT scans, we also incorporated the tumor involvement of this feature into our analyses to study its correlation with LN metastasis. Analyses of pectoralis muscle structure may allow better evaluation of the diagnosis in breast cancer patients and facilitate the optimal selection of therapy (35).For highburden prediction, the relationship between vessels and the mass was also observed with CBBCT due to its unique 3D visual function of the tumor and peri-tumor structure. This feature is similar to the blood flow signal from Doppler ultrasound of the primary tumor, and its association with metastatic LN was reported by a few articles. Our prediction that abundant vasculature within or adjacent to the tumor may be indicative of lymphatic burden, however the feature has no statistical value in this literature. (36).

US-reported abnormal shapes of LNs were also reported in previous publications (37). Wang et al. (38) discovered that HR and Her2 expression are associated with a high-burden LN status. The number of suspicious $L N$ s detected was also related to outcome when using $L N>2$ as the cutoff in the prediction of high burden (38). In this paper, one LN was used as the cutoff for prediction because one abnormal LN on AUS can be predictive of a low burden of $L N s\left(\mathrm{~N}_{1-2}\right)$, as demonstrated by Puri et al. (39) Other US-

dependent characteristics, such as the maximum $L N$ axis and the effacement of the lymphatic hilum, are closely related to a negative LN status (40). The lymphatic hilum is an entrance for lymphatic vessels and nerves. It is in close anatomical proximity to the medulla and cortex. Nevertheless, the predictive value of 
the boundary between the cortex and medulla has rarely been described previously. Few studies have demonstrated that the deformity of the medulla is a predictor of LN metastasis (41).

Above all, our constructed models have advantages for clinical application, and several aspects should be noted. First, we developed models that include parameters that are of practical value and reproducible,. We reassessed variables that were generally evaluated during the process of routine examination to find their association with post-surgical lymphatic outcomes. This type of medical imaging evaluation is time-consuming but still cannot be replaced by computerized methods at present. Noninvasive preoperative parameters that evaluate the risk probabilities of the LN burden in breast cancer will be of value in clinical practice. Our study demonstrated superior discrimination ability for the classification of the LN status using the combination of noninvasive CT and AUS. This association can achieve an equal or higher AUC than noninvasive MRI or US or PET/CT for the evaluation of the LN burden in other papers (14). Second, given that the results obtained from biopsy are regarded as the gold standard and can remarkably improve the prediction performance and are often available after surgery, "bundle/vascular invasion" was not selected for the construction of our models. These factors will introduce some selection bias to the final assessment. Third, we discovered that tumor biology affected the LN outcome because either CBBCT or AUS alone did not provide a better prediction than their combination. Fourth, we provided a novel view of bidirectional feature selection methods that achieved comparative performance in model building compared with other studies.

Despite the advantages offered by the approaches presented herein, there were some limitations to note. First, features of mass-like and non-mass enhancement were not included in the study due to the limited number of patients. Further investigation is needed to study the association between the enhancement type and LN outcome. Second, the morphology and distribution of calcifications play an important role in CBBCT screening compared with in breast MRI and US. However, calcification was not used as a predictor to avoid influencing the model's accuracy and producing bias. Subsequent studies are required to incorporate preoperative imaging features into more specific categories, which may be of greater use for clinical decision making. Third, the robustness and reproducibility of the prediction models needs to be validated in an external cohort, and a larger sample size is needed to optimize the prediction models. Fourth, given that micrometastatic invasion in LNs or early stages of breast cancer can have an inconspicuous appearance on images, they may be underestimated by preoperative examinations. Therefore, further study with a combination of computerized assessments is needed. Fifth, a study showed that a subset of patients with a low burden of SLN had a poor prognosis after simple biopsy. Thus, a more precise evaluation of LNs should be performed in clinical practice in the future (42).

\section{Conclusion}

This study provided a new perspective on incorporating the advanced technology of CBBCT with USreported nodal appearance to develop effective models to improve the preoperative predictive efficacy of the LN burden in T1-2 patients more conveniently and accurately. 


\section{Abbreviations}

CBBCT Cone-beam breast computerized tomography

AUS Axillary ultrasound

SLNB Sentinel lymph node biopsy

ALND Axillary lymph node dissection

LN Lymph node

ER Estrogen receptor

PR Progesterone receptor

IDC Invasive ductal carcinoma

HER2 Human epidermal growth factor receptor 2

ROC Receiver operating characteristic

AUC Area under the curve

DCA Decision curve analysis

HL test Hosmer-Lemeshow tests

$\mathrm{Cl}$ Confidence interval

\section{Declarations}

\section{Ethics approval and consent to participate}

The Institutional Ethics Committee of Sun Yat-sen University Cancer Center (No.B2019-016) approved all experimental protocols of this study and waived informed consent from all participants included in the study prior to the initiation of the study procedure. All methods were carried out in accordance with relevant guidelines and regulations.

\section{Consent for publication}

N/A.

\section{Availability of data and materials}


The database analyzed during the current study are available from the corresponding author on reasonable request. The confidential patient data should not be shared.

\section{Competing interests}

The authors declare no competing interests.

\section{Funding}

This research was supported by the National Key Research and Development Program of China (grant numbers 2107YFC0112600, 2017YFC0112605, 2017YFC0112603 and 2017YFC109304) and Guangdong Medical Science and Technology Research Fund of China (grant number A201805).

\section{Authors' contributions}

$\mathrm{SC}$ and $\mathrm{JL}$ designed the concept of this original research and participated in the data analysis with the professional guidance rendered by PQC. SC drafted the manuscript and all the authors read and approved the final manuscript. TBM provided the assistance regarding graphic preparation. YPW and NH instructed the professional annotation of CT imaging data in the study.

\section{Acknowledgments}

The authors thank Sun Yat-sen University Cancer Center and Guangxi Medical University for technical assistance and provision of the imaging data used in this study.

\section{Conflict of Interest}

The authors declare no competing interests

\section{References}

1. Siegel RL, Miller KD, Jemal A. Cancer statistics, 2020. CA Cancer J Clin. 2020;70(1):7-30.

2. Bevers TB, Helvie M, Bonaccio E, et al. Breast Cancer Screening and Diagnosis, Version 3.2018, NCCN Clinical Practice Guidelines in Oncology. J Natl Compr Canc Netw. 2018;16(11):1362-1389.

3. Chang JM, Leung JWT, Moy L, Ha SM, Moon WK. Axillary Nodal Evaluation in Breast Cancer: State of the Art. Radiology. 2020;295(3):500-515.

4. Huang TW, Kuo KN, Chen $\mathrm{KH}$, et al. Recommendation for axillary lymph node dissection in women with early breast cancer and sentinel node metastasis: A systematic review and meta-analysis of randomized controlled trials using the GRADE system. Int J Surg. 2016;34:73-80.

5. Li CZ, Zhang P, Li RW, Wu CT, Zhang XP, Zhu HC. Axillary lymph node dissection versus sentinel lymph node biopsy alone for early breast cancer with sentinel node metastasis: A meta-analysis. Eur J Surg Oncol. 2015;41(8):958-966. 
6. Giuliano AE, Ballman KV, McCall L, et al. Effect of Axillary Dissection vs No Axillary Dissection on 10Year Overall Survival Among Women With Invasive Breast Cancer and Sentinel Node Metastasis: The ACOSOG Z0011 (Alliance) Randomized Clinical Trial. JAMA. 2017;318(10):918-926.

7. Lee SA, Lee HM, Lee HW, et al. Risk Factors for a False-Negative Result of Sentinel Node Biopsy in Patients with Clinically Node-Negative Breast Cancer. Cancer Res Treat. 2018;50(3):625-633.

8. Andersson Y, Frisell J, Sylvan M, de Boniface J, Bergkvist L. Causes of false-negative sentinel node biopsy in patients with breast cancer. Br J Surg. 2013;100(6):775-783.

9. Yoo TK, Kim SJ, Lee J, et al. A NO Predicting Model for Sentinel Lymph Node Biopsy Omission in Early Breast Cancer Upstaged From Ductal Carcinoma in Situ. Clin Breast Cancer. 2020;20(3):e281e289.

10. Yoo J, Kim BS, Yoon HJ. Predictive value of primary tumor parameters using 18F-FDG PET/CT for occult lymph node metastasis in breast cancer with clinically negative axillary lymph node. Ann Nucl Med. 2018;32(9):642-648.

11. Sun Q, Lin X, Zhao Y, et al. Deep Learning vs. Radiomics for Predicting Axillary Lymph Node Metastasis of Breast Cancer Using Ultrasound Images: Don't Forget the Peritumoral Region. Front Oncol. 2020;10:53. Published 2020 Jan 31.

12. Wienbeck S, Fischer U, Luftner-Nagel S, Lotz J, Uhlig J. Contrast-enhanced cone-beam breast-CT (CBBCT): clinical performance compared to mammography and MRI. Eur Radiol. 2018;28(9):37313741.

13. Wienbeck S, Uhlig J, Luftner-Nagel S, Zapf A, Surov A, von Fintel E, et al. The role of cone-beam breast-CT for breast cancer detection relative to breast density. Eur Radiol (2017) 27:5185-95.

14. Uhlig J, Uhlig A, Biggemann L, Fischer U, Lotz J, Wienbeck S. Diagnostic accuracy of cone-beam breast computed tomography: a systematic review and diagnostic meta-analysis. Eur Radiol. 2019;29(3):1194-1202.

15. Wienbeck S, Lotz J, Fischer U. Review of clinical studies and first clinical experiences with a commercially available cone-beam breast CT in Europe. Clin Imaging. 2017;42:50-59.

16. Li H, Yin L, He N, et al. Comparison of comfort between cone beam breast computed tomography and digital mammography. Eur J Radiol. 2019;120:108674.

17. Li J, Ma W, Jiang X, et al. Development and Validation of Nomograms Predictive of Axillary Nodal Status to Guide Surgical Decision-Making in Early-Stage Breast Cancer. J Cancer. 2019;10(5):12631274. Published 2019 Jan 29.

18. Jackson RS, Mylander C, Rosman M, et al. Normal Axillary Ultrasound Excludes Heavy Nodal Disease Burden in Patients with Breast Cancer. Ann Surg Oncol. 2015;22(10):3289-3295.

19. Luo Y, Zhao C, Gao Y, et al. Predicting Axillary Lymph Node Status With a Nomogram Based on Breast Lesion Ultrasound Features: Performance in N1 Breast Cancer Patients. Front Oncol. 2020;10:581321. Published 2020 Oct 27.

20. Scaranelo AM, Eiada R, Jacks LM, Kulkarni SR, Crystal P. Accuracy of unenhanced MR imaging in the detection of axillary lymph node metastasis: study of reproducibility and reliability. Radiology. 
2012;262(2):425-434.

21. Yu FH, Wang JX, Ye XH, Deng J, Hang J, Yang B. Ultrasound-based radiomics nomogram: A potential biomarker to predict axillary lymph node metastasis in early-stage invasive breast cancer. Eur $J$ Radiol. 2019;119:108658.

22. Long HV, Son LH, Khari M, et al. A New Approach for Construction of Geodemographic Segmentation Model and Prediction Analysis. Comput Intell Neurosci. 2019;2019:9252837. Published 2019 May 13.

23. Fishbein DH, Novak SP, Ridenour TA, Thornburg V, Hammond J, Brown J. Neurocognitive Characteristics of Early Marijuana Use Initiation in Adolescents: A Signature Mapping Analysis. $J$ Stud Alcohol Drugs. 2016;77(3):431-440.

24. Prionas ND, Lindfors KK, Ray S, et al. Contrast-enhanced dedicated breast CT: initial clinical experience. Radiology. 2010;256(3):714-723.

25. Campbell EJ, Tesson M, Doogan F, Mohammed ZMA, Mallon E, Edwards J. The combined endocrine receptor in breast cancer, a novel approach to traditional hormone receptor interpretation and a better discriminator of outcome than ER and PR alone. Br J Cancer. 2016;115(8):967-973.

26. Yoshihara E, Smeets A, Laenen A, et al. Predictors of axillary lymph node metastases in early breast cancer and their applicability in clinical practice. Breast. 2013;22(3):357-361. doi:10.1016/j.breast.2012.09.003

27. Piñero-Madrona A, Ruiz-Merino $G$, Bernet $L$, et al. Tumoral load quantification of positive sentinel lymph nodes in breast cancer to predict more than two involved nodes. Breast. 2014;23(6):859-864.

28. Cabioglu N, Ozmen $\mathrm{V}$, Kaya $\mathrm{H}$, et al. Increased lymph node positivity in multifocal and multicentric breast cancer. J Am Coll Surg. 2009;208(1):67-74.

29. Weissenbacher TM, Zschage M, Janni W, et al. Multicentric and multifocal versus unifocal breast cancer: is the tumor-node-metastasis classification justified?. Breast Cancer Res Treat. 2010;122(1):27-34.

30. Frey JD, Salibian AA, Lee J, et al. Oncologic Trends, Outcomes, and Risk Factors for Locoregional Recurrence: An Analysis of Tumor-to-Nipple Distance and Critical Factors in Therapeutic NippleSparing Mastectomy. Plast Reconstr Surg. 2019;143(6):1575-1585.

31. Ansari B, Morton MJ, Adamczyk DL, et al. Distance of breast cancer from the skin and nipple impacts axillary nodal metastases. Ann Surg Oncol. 2011;18(11):3174-3180.

32. Torstenson T, Shah-Khan MG, Hoskin TL, et al. Novel factors to improve prediction of nodal positivity in patients with clinical T1/T2 breast cancers. Ann Surg Oncol. 2013;20(10):3286-3293.

33. Zhang Y, Li J, Fan Y, et al. Risk factors for axillary lymph node metastases in clinical stage T12NOMO breast cancer patients. Medicine (Baltimore). 2019;98(40):e17481.

34. Rossi F, Valdora F, Barabino E, Calabrese M, Tagliafico AS. Muscle mass estimation on breast magnetic resonance imaging in breast cancer patients: comparison between psoas muscle area on computer tomography and pectoralis muscle area on MRI. Eur Radiol. 2019;29(2):494-500. 
35. Morris EA, Schwartz LH, Drotman MB, et al. Evaluation of pectoralis major muscle in patients with posterior breast tumors on breast MR images: early experience. Radiology. 2000;214(1):67-72.

36. Dialani V, Gaur S, Mehta TS, et al. Prediction of Low versus High Recurrence Scores in Estrogen Receptor-Positive, Lymph Node-Negative Invasive Breast Cancer on the Basis of RadiologicPathologic Features: Comparison with Oncotype DX Test Recurrence Scores. Radiology. 2016;280(2):370-378.

37. Moore A, Hester M, Nam MW, et al. Distinct lymph nodal sonographic characteristics in breast cancer patients at high risk for axillary metastases correlate with the final axillary stage. Br J Radiol. 2008;81(968):630-636.

38. Wang X, Chen L, Sun Y, Zhang B. Evaluation of axillary lymph node metastasis burden by preoperative ultrasound in early-stage breast cancer with needle biopsy-proven metastasis. Clin Trans/ Oncol. 2020;22(4):468-473.

39. Puri S, Sharma N, Newcombe RG, et al. Axillary tumour burden in women with one abnormal node on ultrasound compared to women with multiple abnormal nodes. Clin Radiol. 2018;73(4):391-395.

40. Han P, Yang H, Liu M, et al. Lymph Node Predictive Model with in Vitro Ultrasound Features for Breast Cancer Lymph Node Metastasis. Ultrasound Med Biol. 2020;46(6):1395-1402.

41. Wang G, Li X, Li L, et al. Clinical value of ultrasonic imaging in diagnosis of hypopharyngeal cancer with cervical lymph node metastasis. Oncol Lett. 2019;18(6):5917-5922.

42. Yang $X, M a X$, Yang W, Shui R. Clinical significance of extranodal extension in sentinel lymph node positive breast cancer. Sci Rep. 2020;10(1):14684. Published 2020 Sep 7.

\section{Tables}


TABLE 1 | Clinicopathological characteristics of patients in training and validation cohorts. Training cohort $(n=271) \quad$ Validation cohort $(n=116) \quad p$-value

\begin{tabular}{lcc} 
Age, mean \pm SD, years & $50.4 \pm 10.0$ & $48.3 \pm 9.0$ \\
\hline Side & & \\
\hline left & $144 \rrbracket 53.1 \rrbracket$ & $69 \llbracket 59.5 \rrbracket$ \\
right & $127 \rrbracket 46.9 \rrbracket$ & $47 \rrbracket 40.5 \rrbracket$
\end{tabular}

Histological type

0.008

pure IDC

$89 \rrbracket 32.8 \rrbracket$

57囚49.1凶

mixed IDC

$169862.4 \rrbracket$

$53 \rrbracket 45.7 \rrbracket$

other special types of $\mathrm{BC}$

$13 \llbracket 4.8 \rrbracket$

$6 \rrbracket 5.2 \rrbracket$

Pathologic grade

0.924

Low

$5 \otimes 1.8 \rrbracket$

$3 \otimes 2.6 \rrbracket$

Moderate

145囚53.5区

63囚54.3)

High

109ه40.3》

44(37.9)

absent

$12 \llbracket 4.4 \rrbracket$

6(5.2)

ER/PR

0.075

positive

42(15.5)

10(8.6)

negative

229(84.5)

106(91.4)

HER2 overexpression

0.686

with

211(77.9)

93(80.2)

without

60(22.1)

23(19.8)

Ki-67 status

0.184

low

75(27.7)

40(34.5)

high

196(72.3)

76(65.5)

Bundle invasion

0.886

absent

222(81.9)

94(81.0)

present

49(18.1)

22(19.0)

Vascular invasion

absent

182(67.2)

0.906

(absent

79(68.1) 
TABLE 2 | Univariate Regression Analysis of Preoperative Data

\begin{tabular}{|c|c|c|c|c|}
\hline & Model one & & Model two & \\
\hline & $\mathrm{N}$ - versus $\mathrm{N}+$ & & $N \otimes 3$ versus $N \geq 3$ & \\
\hline & OR & p-value & OR & $\mathrm{p}$-value \\
\hline Maximum diameter (mm) & $\begin{array}{l}1.053(1.027 \\
1.081)\end{array}$ & $<0.001$ & $1.051(1.024,1.079)$ & $<0.001$ \\
\hline Distance to nipple (mm) & $\begin{array}{l}0.982(0.970 \\
0.994)\end{array}$ & 0.003 & $0.981(0.965,0.996)$ & 0.015 \\
\hline lesion number & & 0.162 & & 0.001 \\
\hline one lesion & reference & & reference & \\
\hline More than one lesion & $\begin{array}{l}\text { 1.442(0.861, } \\
2.411)\end{array}$ & & $2.780(1.493,5.177)$ & \\
\hline Thickened or sunken skin & & $<0.001$ & & 0.018 \\
\hline absent & reference & & reference & \\
\hline present & $\begin{array}{l}3.419(1.753 \\
6.669)\end{array}$ & & $2.396(1.160,4.951)$ & \\
\hline Subcutaneous fat space & & $<0.001$ & & $<0.001$ \\
\hline clear & reference & & reference & \\
\hline misty & $\begin{array}{l}2.996(1.756 \\
5.111)\end{array}$ & & $5.375(2.416,11.958)$ & \\
\hline Invasion of pectoralis major & & 0.002 & & 0.020 \\
\hline absent & reference & & reference & \\
\hline present & $\begin{array}{l}6.349(2.009 \\
20.059)\end{array}$ & & $3.341(1.206,9.256)$ & \\
\hline number of LN & & 0.003 & & 0.003 \\
\hline none & reference & & reference & \\
\hline one & $\begin{array}{l}0.339(0.182, \\
0.631)\end{array}$ & & $0.121(0.036,0.402)$ & \\
\hline more than one LN & $\begin{array}{l}0.788(0.183 \\
3.393)\end{array}$ & & $1.007(0.196,5.165)$ & \\
\hline maximum LN length $(\mathrm{mm})$ & $\begin{array}{l}1.046(1.016 \\
1.077)\end{array}$ & 0.002 & $1.065(1.027,1.105)$ & 0.001 \\
\hline maximum LN axis (mm) & $\begin{array}{l}\text { 1.223(1.134, } \\
1.319)\end{array}$ & $<0.001$ & $1.345(1.205,1.502)$ & $<0.001$ \\
\hline
\end{tabular}




\begin{tabular}{|c|c|c|c|c|}
\hline Lymph node's shape & & $<0.001$ & & $<0.001$ \\
\hline oval & reference & & reference & \\
\hline round & $\begin{array}{l}10.039(1.210,82 . \\
278)\end{array}$ & & $24.585(2.940,205.608)$ & \\
\hline absent & $\begin{array}{l}0.370(0.199 \\
0.690)\end{array}$ & & $0.140(0.042,0.469)$ & \\
\hline Blood flow type & & 0.002 & & 0.045 \\
\hline undetected & reference & & reference & \\
\hline portal type & $\begin{array}{l}1.596(0.890 \\
2.860)\end{array}$ & & $1.477(0.725,3.009)$ & \\
\hline mixed peripheral type & $\begin{array}{l}5.614(2.072 \\
15.213)\end{array}$ & & $3.310(1.260,8.696)$ & \\
\hline $\begin{array}{l}\text { Boundary between cortex and } \\
\text { medulla }\end{array}$ & & $<0.001$ & & $<0.001$ \\
\hline distinct & reference & & reference & \\
\hline indistinct & $\begin{array}{l}10.346(4.986 \\
21.471)\end{array}$ & & $14.688(7.150,30.172)$ & \\
\hline $\begin{array}{l}\text { Relationships between vessel } \\
\text { and mass }\end{array}$ & & 0.178 & & 0.045 \\
\hline Inside mass & reference & & reference & \\
\hline Outside mass & $\begin{array}{l}1.407(0.856 \\
2.312)\end{array}$ & & $1.880(1.013,3.488)$ & \\
\hline Bundle invasion & & 0.003 & & 0.022 \\
\hline absent & reference & & reference & \\
\hline present & $\begin{array}{l}2.557(1.363 \\
4.797)\end{array}$ & & $2.279(1.127,4.611)$ & \\
\hline Vascular invasion & & $<0.001$ & & $<0.001$ \\
\hline absent & reference & & reference & \\
\hline present & $\begin{array}{l}12.957(7.067 \\
23.754)\end{array}$ & & $7.562(3.843,14.882)$ & \\
\hline
\end{tabular}


TABLE 3 | Multivariable Regression Analysis of Preoperative Data Model one

Model two

\begin{tabular}{|c|c|c|c|c|}
\hline $\mathrm{N}$ - versus N+ & versus $\mathrm{N} \rrbracket 3$ & & & \\
\hline - & OR & p-value & OR & $\mathrm{p}$-value \\
\hline (Reference) & 1 & & 1 & \\
\hline Maximum diameter (mm) & $1.024(0.991,1.056)$ & 0.023 & - & 0.022 \\
\hline Distance to nipple (mm) & $0.979(0.961,0.997 \rrbracket$ & 0.034 & $0.923(0.856,0.991)$ & 0.006 \\
\hline Number of LN & & / & & 0.039 \\
\hline solitary & l & & $9.417(1.335,96.526)$ & \\
\hline More than one & l & & $0.978(0.886,1.080)$ & \\
\hline Maximum $L N$ axis & $1.341(1.112,1.683)$ & 0.006 & l & / \\
\hline LN shape & & 0.013 & & 0.022 \\
\hline Oval & $2.917 \rrbracket 1.326 \rrbracket 7.157 \rrbracket$ & & $13.726(1.895,161.976)$ & \\
\hline irrugular & $5.169(0.960,31.84)$ & & $1.698(0.220,13.102)$ & \\
\hline $\begin{array}{l}\text { Boundary between cortex } \\
\text { and medulla }\end{array}$ & & $<0.001$ & & $<0.001$ \\
\hline misty & $7.654(2.746,23.104)$ & & $6.334(2.359,17.844)$ & \\
\hline Subcutaneous fat space & & 0.047 & & 0.003 \\
\hline misty & $2.146(1.019,4.650)$ & & $5.012(1.607,19.362)$ & \\
\hline $\begin{array}{l}\text { Invasion of pectoralis } \\
\text { major }\end{array}$ & & 0.002 & & / \\
\hline present & $12.194(2.528,64.457)$ & & l & \\
\hline Bundle invasion & & 0.007 & & I \\
\hline present & $3.424(1.396,8.542)$ & & I & \\
\hline Vascular invasion & & $<0.001$ & & $<0.001$ \\
\hline present & $12.691(5.923,9.113)$ & & $7.234(2.899,9.494)$ & \\
\hline
\end{tabular}

\section{Figures}




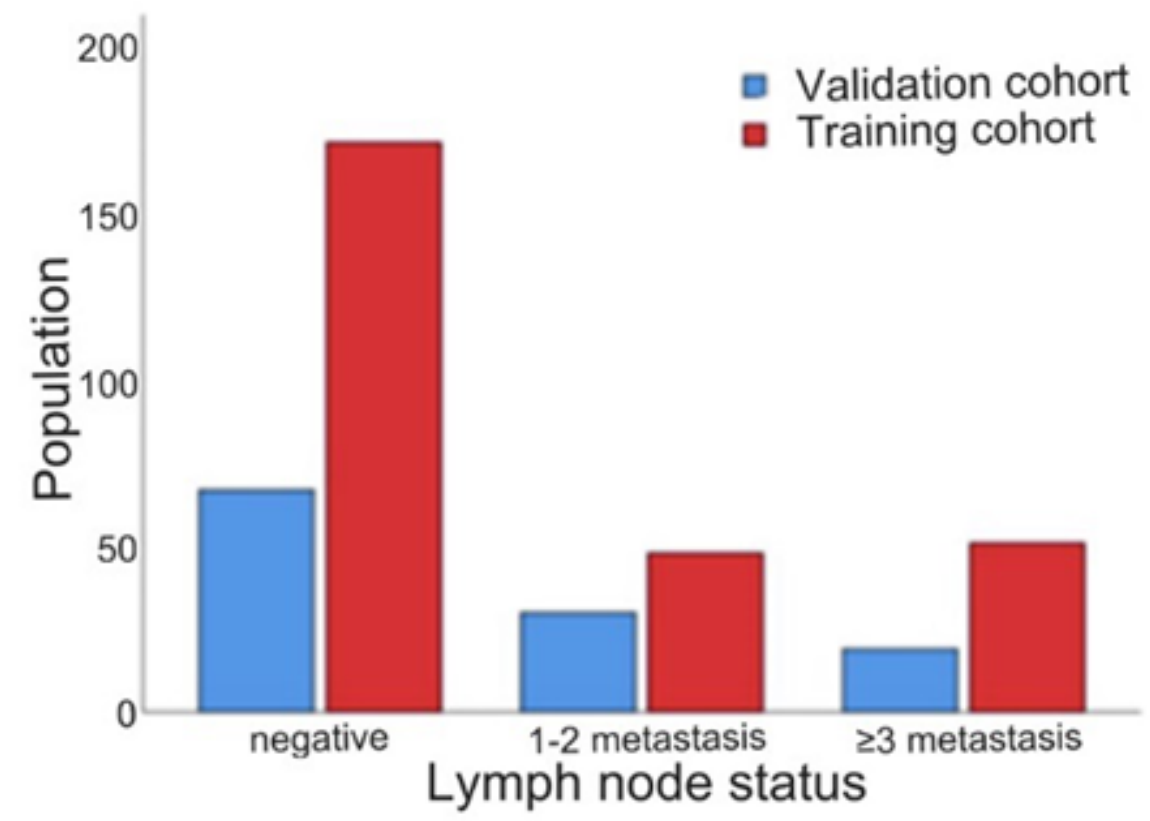

Figure 1

Percentages of the population in both the primary and validation cohorts for the stratification of LN burden.

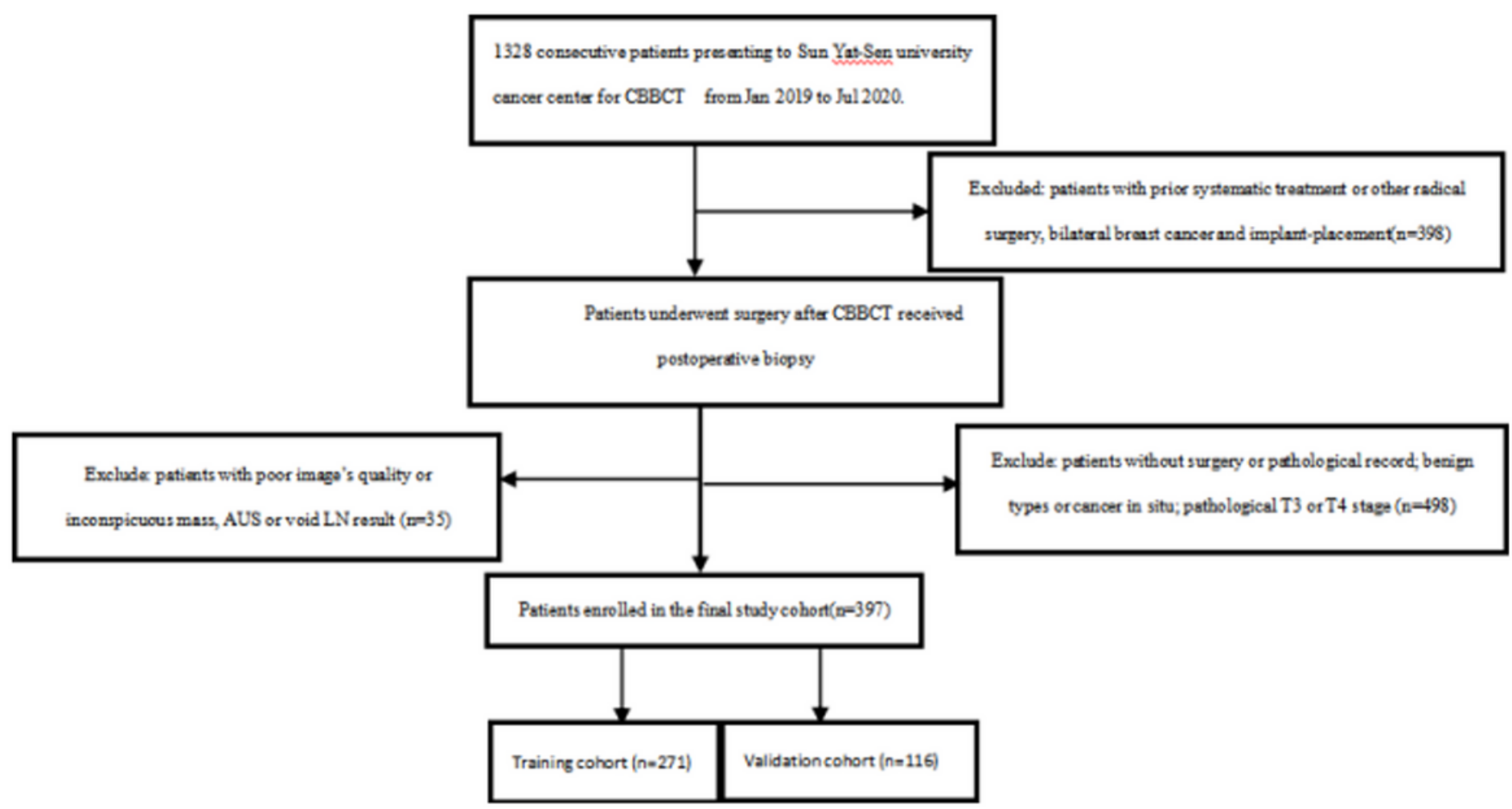

Figure 2 
Flowchart of study population recruitment. ALND = axillary lymph node dissection, $S L N B=$ sentinel lymph node biopsy.
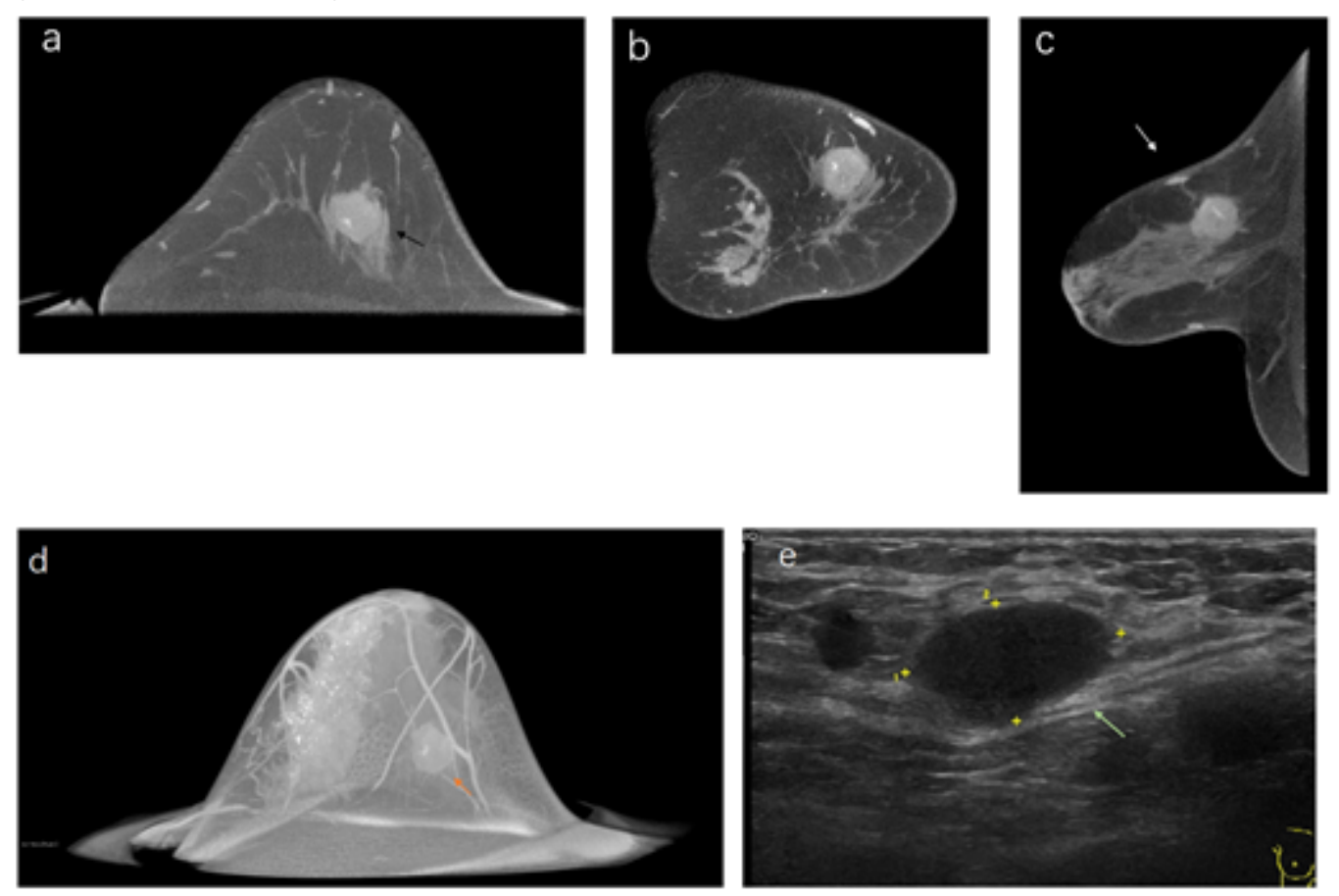

\section{Figure 3}

Right-side breast lesion in a 65-year-old asymptomatic woman (invasive ductal cancer, grade 3). (A,B) Plain CBBCT scan showed an irregular high-density mass with a lobular margin; a single group of calcifications (black arrow) were present inside the mass with fine pleomorphic morphology. (C) The signal was heterogeneously elevated on 120 s contrast-enhanced CT images (white arrow). (D) The threedimensional reconstruction of tumor's primary morphology and its relationship with peritumor vessels (red arrow) are presented with CBBCT dedicated software. (E)Axillary ultrasound clearly showed an asymmetric lymph node with the absence of a hilum. Its boundary between medulla and cortex is unclear(green arrow). 
A

Points

Maximum diameter $(\mathrm{mm})$

Distance to nipple (mm)

Maximum LN axis (mm)

Boundary between cortex and medulla

LN shape

Subcutaneous fat space

Invasion of pectoralis major

Total Points

Linear Predictor

Risk

B

Points

Maximum diameter $(\mathrm{mm})$

Distance to nipple (mm)

Number of LN

Boundary between cortex and medulla

LN shape

Subcutaneous fat space

Total Points

Linear Predictor

Risk

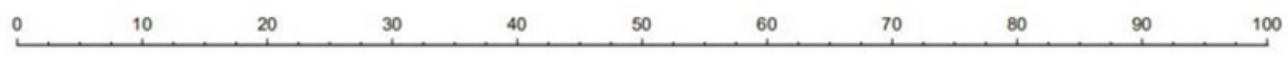

$\begin{array}{llllllllllll}0 & 10 & 20 & 30 & 40 & 50 & 60 & 70 & 80 & 90 & 100\end{array}$

$\begin{array}{rllllllllllllll}120 & 100 & 90 & 80 & 70 & 60 & 50 & 40 & 30 & 20 & 10 & 0\end{array}$

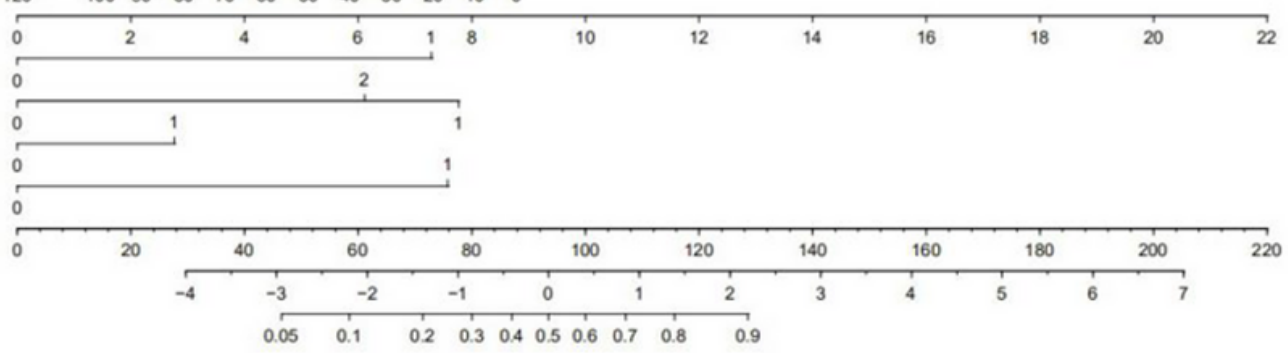

\begin{tabular}{l}
10 \\
0.5 \\
\hline
\end{tabular}

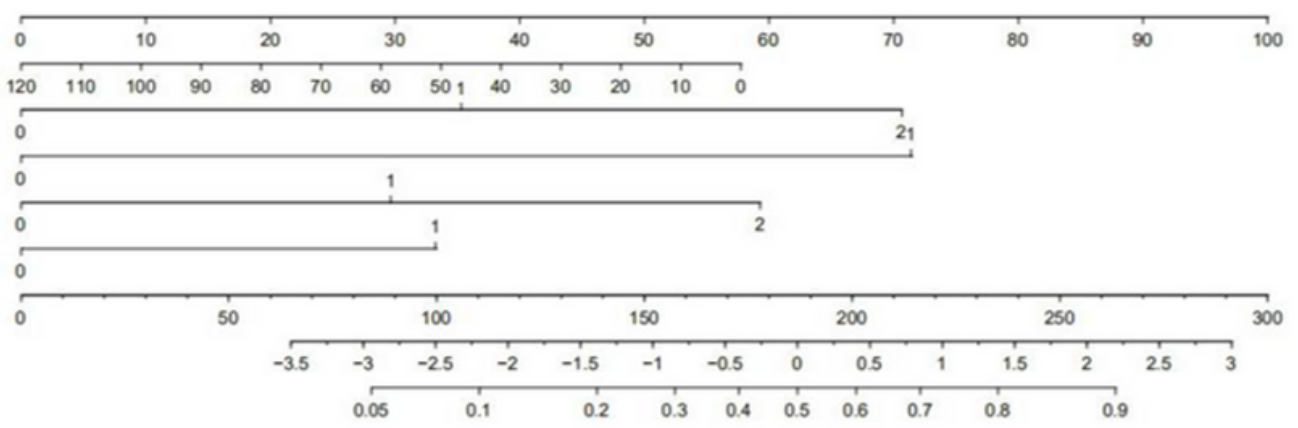

Figure 4

Two independent models were developed based on the radiographic interpretation of CBBCT and AUS to grade the lymph node burden. Nomograms are presented as scoring tools to predict $(A)$ disease-free axilla (N-) vs. any nodal metastasis $(\mathrm{N}+)$. (B) Nomogram predicting low metastatic burden involving less than three positive nodes $(\mathrm{N}<3)$ vs. heavy-burden axillary diseases involving at least 3 positive nodes $(\mathrm{N} \geq 3)$. 

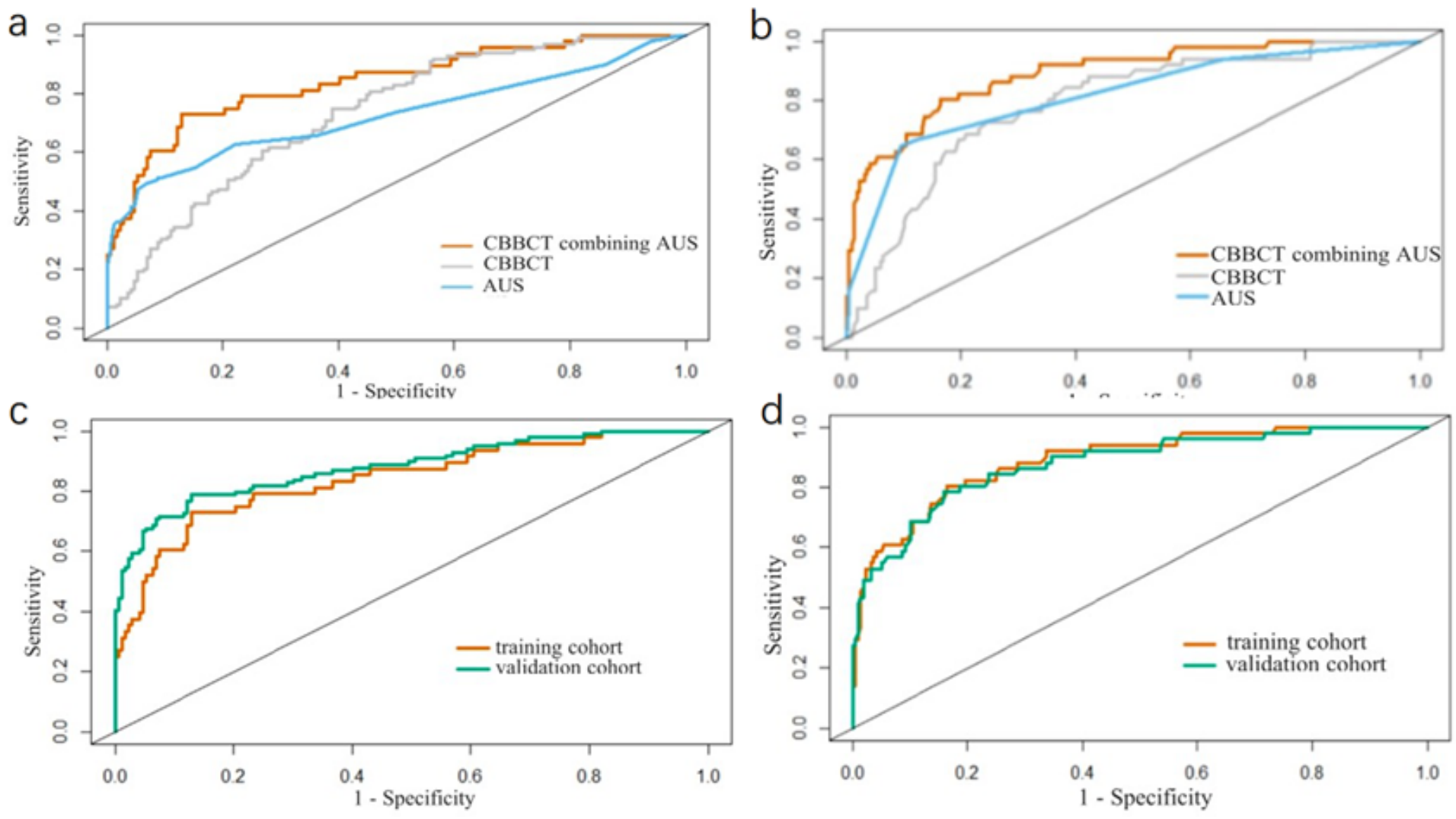

Figure 5

Distribution of ROCs for prediction models. ROCs of the CBBCT combining AUS, CBBCT and AUS for independent prediction in the model 1 (A) and model 2 (B); ROC curves of the primary and validation set for model 1 (C) and model 2 (D). 


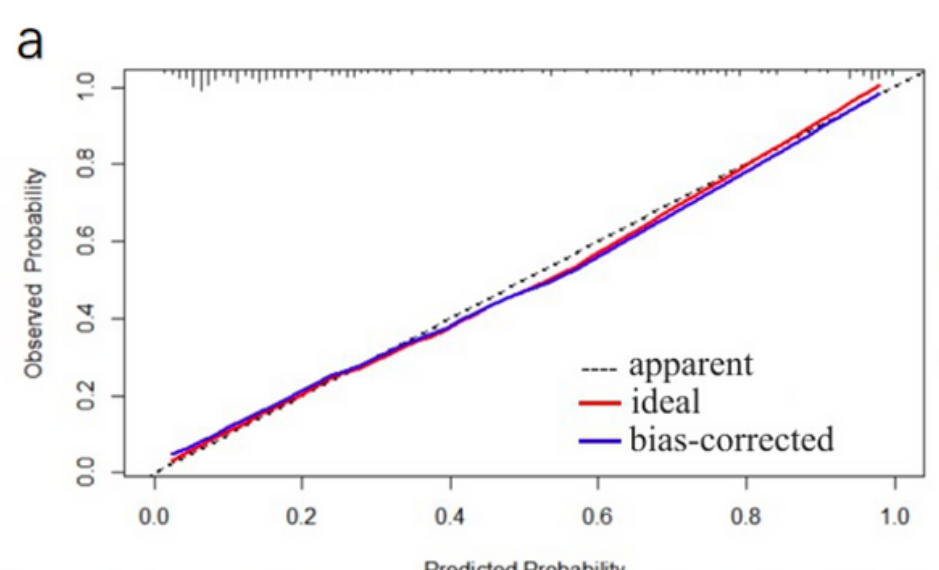

b
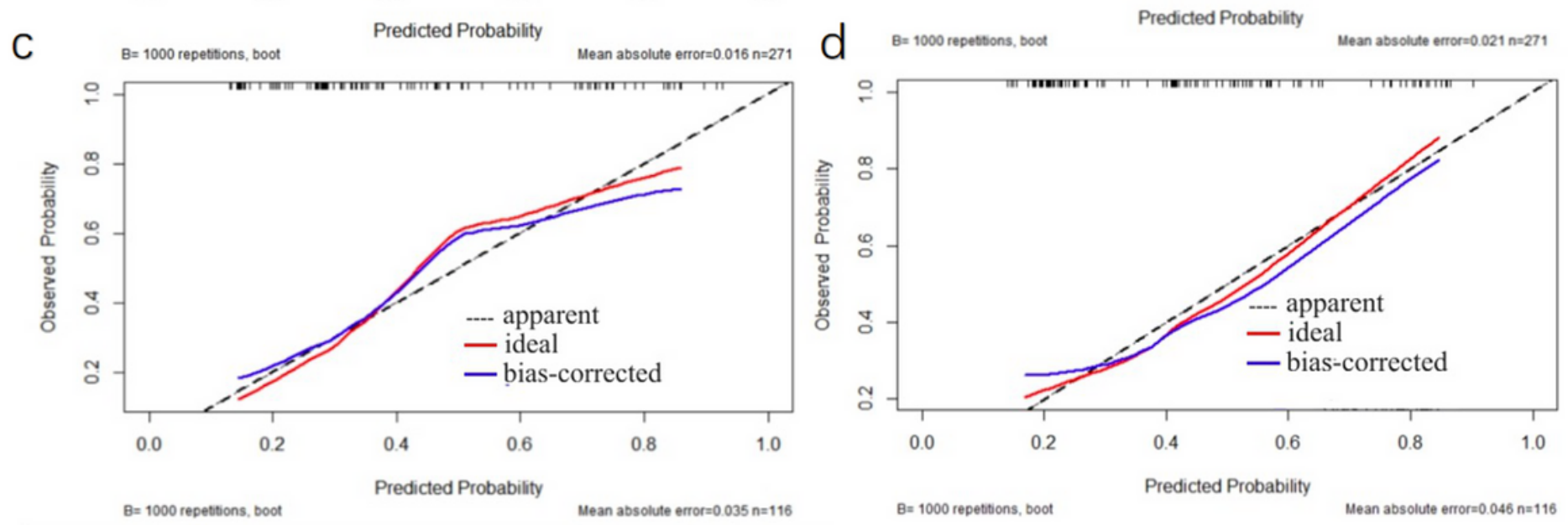

\section{Figure 6}

Calibration curves of the first model in the primary (A) and validation (C) sets. Calibration of the second model in both the primary cohort (B) and validation cohort (D). Calibration curves were plotted after internal validation by bootstrapping (repetition $=1000$ ). The dotted diagonal line represents the reference. 


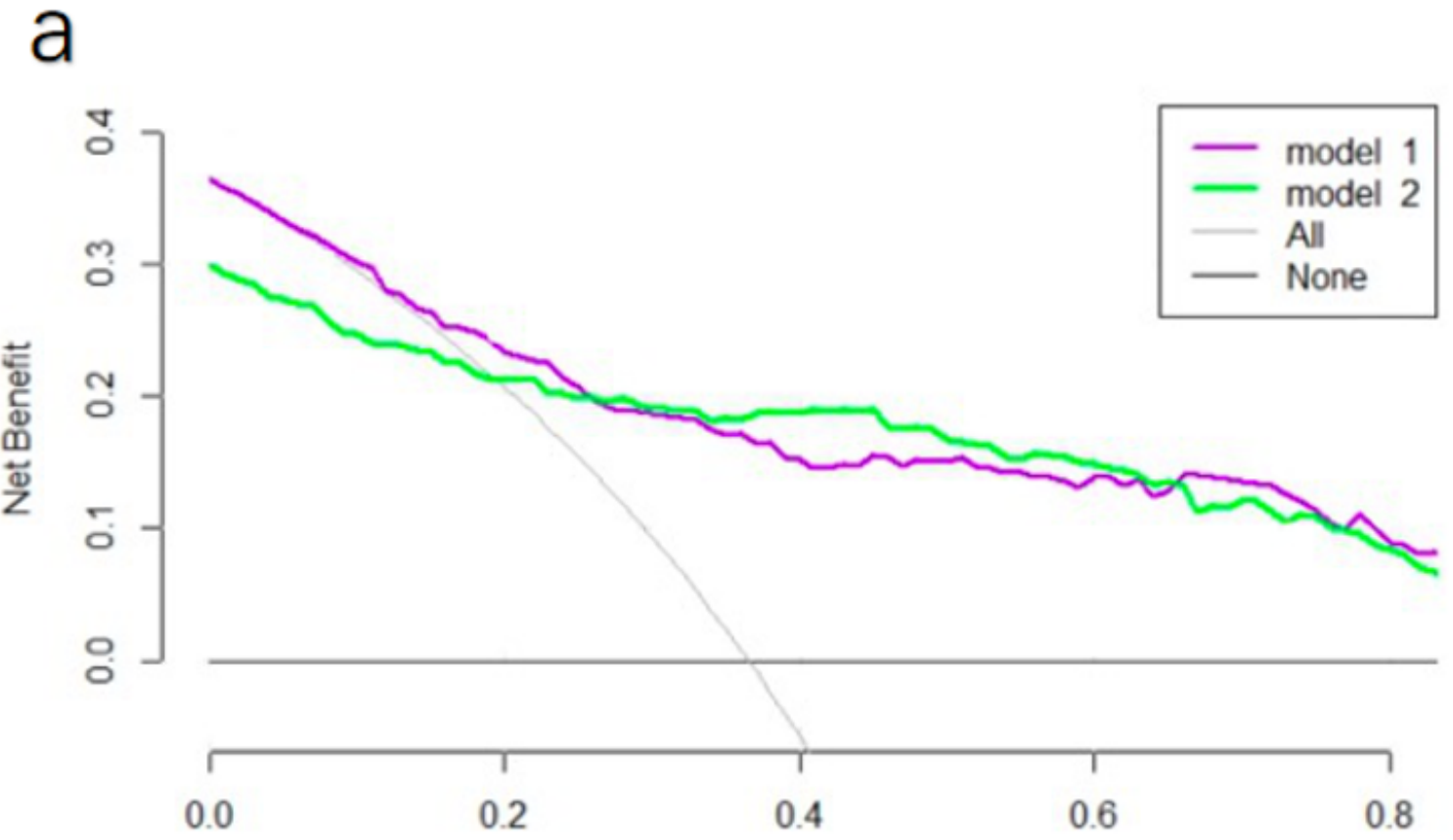

High Risk Threshold

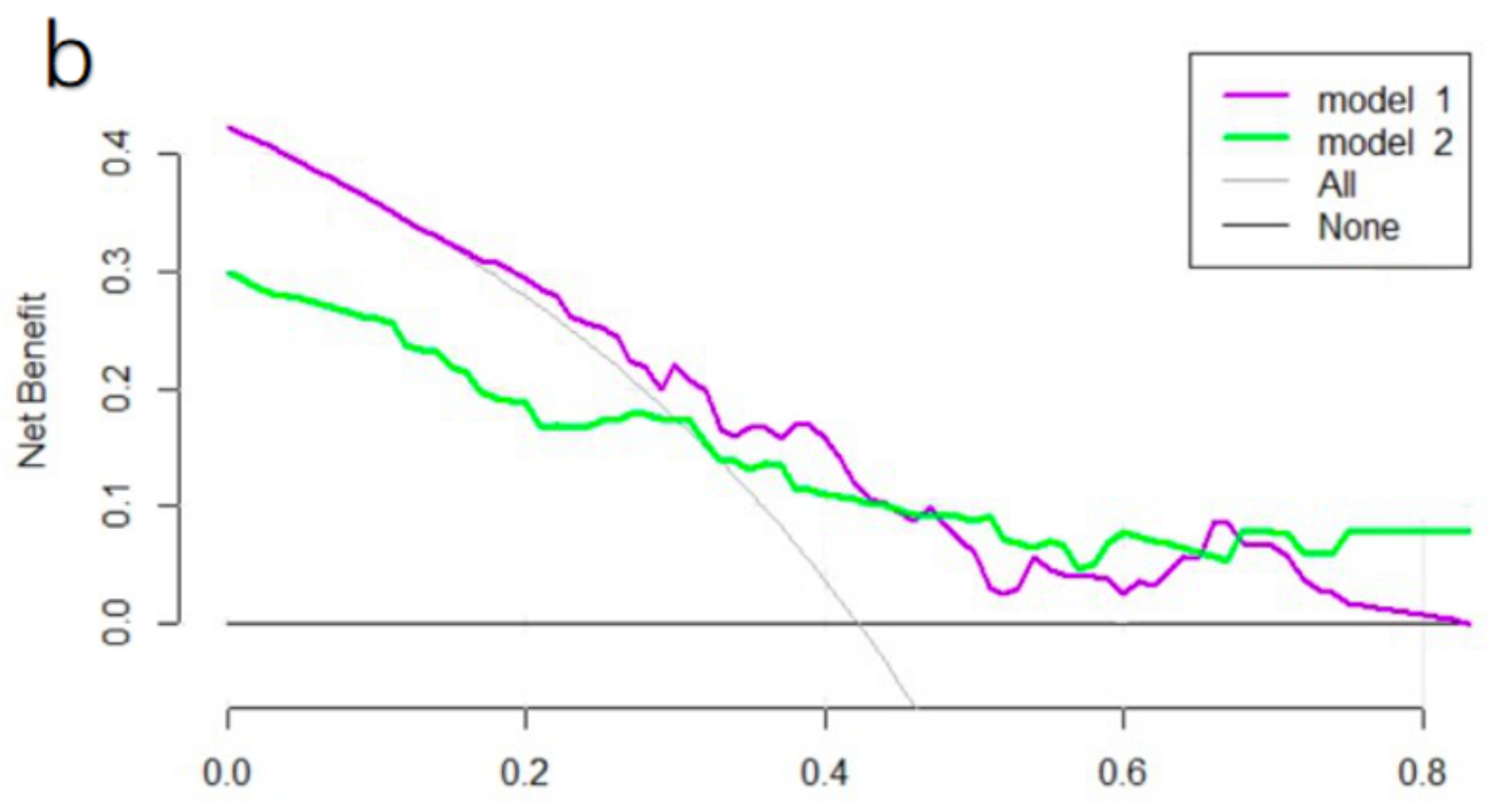

High Risk Threshold

\section{Figure 7}

Decision curve analysis of both combined models in the primary $(A)$ and validation cohorts (B). Lymph node metastasis; (model 1=prediction of the existence of metastasis; model 2=prediction of the LN metastatic burden). Two additional lines in this picture represent two extreme events. The horizontal line indicates that all samples had no metastasis in the models, and the net benefit was 0 . The thin line indicates that all patients were ALN-positive and warrant intervention. 


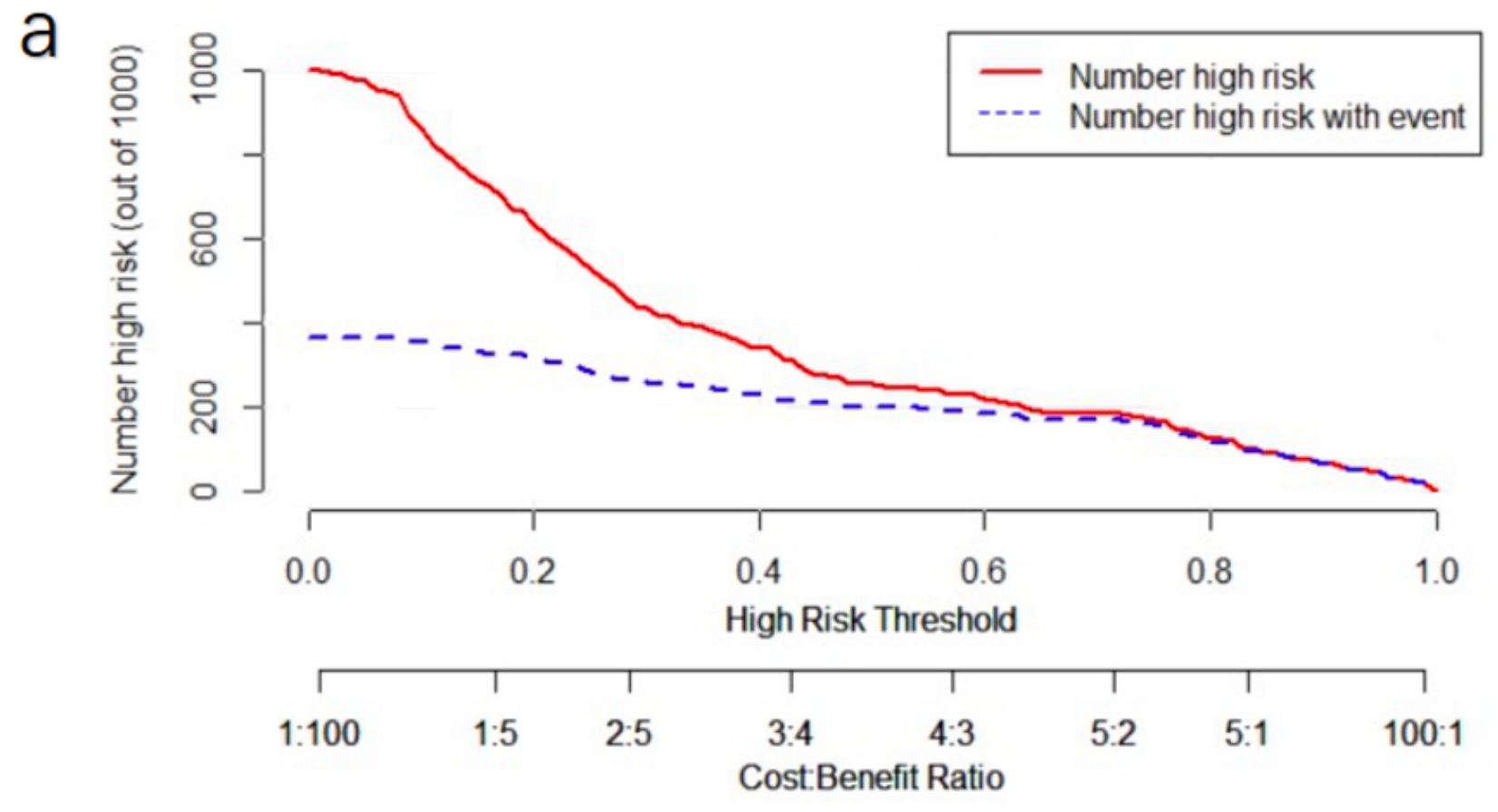

b

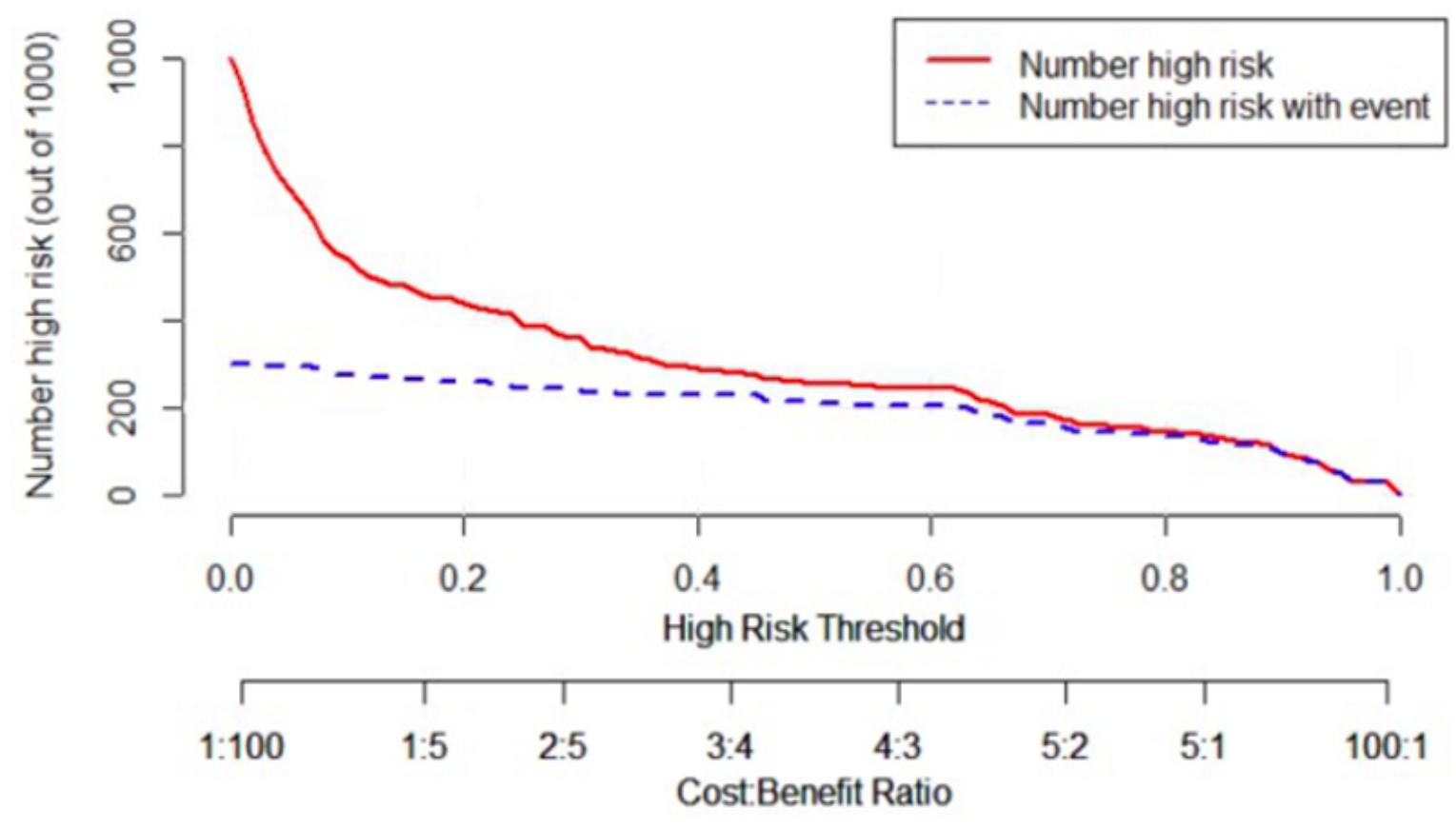

Figure 8

Clinical impact curve analysis of model $1(\mathrm{~A})$ and model 2 (B). The Y-axis shows the number of truepositive high-risk patients (blue dotted line) and the number of high-risk patients with events (red solid line) based on the corresponding high-risk threshold

\section{Supplementary Files}


This is a list of supplementary files associated with this preprint. Click to download.

- Supplementary.docx 